\title{
GEO Satellite Feeder links and Terrestrial Full-Duplex Small Cells: A case for Coexistence
}

\author{
Bhavani Shankar Mysore R*, Sina Maleki*, Gan Zheng*†, Adegbenga Awoseyila ${ }^{\ddagger}$, Barry Evans ${ }^{\ddagger}$ and Björn Ottersten * \\ * Interdisciplinary Centre for Security, Reliability and Trust, University of Luxembourg, L-2721 Luxembourg \\ $\dagger$ School of Computer Science and Electronic Engineering, University of Essex, Colchester, UK, CO4 3SQ \\ $\ddagger$ Institute for Communications Systems, University of Surrey, Guildford, GU2 7XH \\ Email: \{bhavani.shankar, sina.maleki, bjorn.ottersten\}@uni.lu, ganzheng@essex.ac.uk, \{a.awoseyila, b.evans\}@ surrey.ac.uk
}

\begin{abstract}
The demand for wider bandwidths has motivated the need for wireless systems to migrate to higher frequency bands. In line with this trend is an envisaged deployment of Kaband (or mmWave) cellular infrastructure. Further, to improve the spectral efficiency, developing full-duplex radio transceivers is gaining momentum. In view of this move, the paper proposes the possibility of reusing the satellite feeder uplink band in the full-duplex small cells. The motivation for such a reuse is two-fold :(a) there is virtually no interference from the small cells to the incumbent in-orbit satellite receiver, and (b) directive feeder antennas, with possibly additional isolation and processing causing negligible interference to the small cells. The presented interference analysis clearly supports the proposed coexistence.
\end{abstract}

\section{INTRODUCTION}

With enhanced connectivity, new services and applications, there has been an increasing demand for throughput from the terrestrial and satellite systems alike. This has led to the acquisition of exclusive frequencies as well as an exploration of co-existence of the two systems. Dynamic or uncoordinated spectrum utilization employing cognitive radios enable such a co-existence by letting the cognitive user utilize the spectrum of the incumbent user without prior regulatory coordination and waiving right of interference protection, conditioned on not imposing harmful interference to the incumbent user [1]. The idea of coexistence of satellite networks with other services is considered in a number of recent works [1] [6]. These works typically deal with coexistence scenarios involving satellite downlink/ uplink and terrestrial cellular networks, [2], [6] or satellite uplink with downlink of fixedsatellite-services (FSS) [3].

In this work, we consider a dynamic access scenario where the feeder uplink of a Geostationary (GEO) satellite is the incumbent service and the envisaged terrestrial small cells serve as the secondary users [7]. Such feeder links in the licensed Ka-band, like the broadcasting-satellite-service (BSS) uplinks in $17-40 \mathrm{GHz}$, use highly directive antennas thereby encouraging spatial reuse of the frequencies by cognitive users. Further, short range small cells ${ }^{1}$ planned to operate around the Ka-band, employ low-power transmissions [7]; such transmissions can not reach the distant in-orbit GEO

\footnotetext{
${ }^{1}$ Significance of small cells in providing improved cellular coverage capacity and applications for homes and enterprises as well as metropolitan and rural areas is well-known [8].
}

satellite receiver and thus produce no interference to the satellite.

The feeder uplink band can be used for either the uplink or the downlink in the small cell. This motivates the use of full-duplex relaying (FDR) where the small cell base station receives and transmits simultaneously in the same frequency band. FDR has received a noticeable attention recently for its potential to double the spectral efficiency [9]. The use of FDR within the small cell reduces the bandwidth used per small cell and allows for the deployment of a large number of small cells reusing feeder link frequencies. Such a deployment further enhances the efficiency of the terrestrial communication [7].

It is well known that the bottleneck in FDR is the selfinterference (SI) due to signal leakage from the output of the relay onto the signal being received [10]. The large power differential between the SI and the received signal of interest normally saturates the receiver front-end of the relay, thereby necessitating a significant cancellation of SI [10], [11], [12]. Several seminal works on SI cancellation, for e.g., [13] - [15], suppress SI to the noise level. Use of full-duplex enhancements in LTE type small cells has been pursued in [9] where several scenarios are described and the achievable gains are listed. Feasibility of FDR in satellites has been explored in [16].

The purpose of the paper is to evaluate the benefits and challenges of the co-existence of GEO satellite feeder links (e.g., BSS links) and FDR small cells. Following an analytical approach, various interference terms affecting the small cell transmissions are modelled (interference from the feeder link, SI) and a closed-form expression for the resulting Signal to Interference plus Noise ratio (SINR) at the small cell receiver is derived. Impact of various interference components on the SINR is evaluated and a feasibility analysis of the proposed system is presented. The benefits of reuse in general and the employment of full-duplex in particular are then highlighted.

The reminder of the paper is organized as follows: Section II details the scenario and describes the signal model, Section III presents a detailed analysis of the resulting SINR at the receiver, Section IV presents the numerical evaluations along with a discussion on the results while Section V concludes the paper. 


\section{Scenario AND Signal Model}

The paper proposes the reuse of the GEO feeder uplink (for e.g, BSS uplink) frequencies within the small cells. Key attributes of the scenario - low powered small cells and highly directive feeder link antennas - can be exploited to restrict the resulting co-channel interference between the satellite system and small cells within preset thresholds.

\section{A. Full-Duplex Relaying in Small cells}

Small cells, the secondary users in the envisaged scenario, refer to a family of coverage regions having cell radii extending from 10 meters to a 1-2 kilometers, transmit powers varying from $0 \mathrm{dBm}$ to $40 \mathrm{dBm}$, the number of users served per base station (BS) ranging from a couple to hundreds and deployments in different frequency bands. The reader is kindly referred to [7], [17] for details. In this paper, we refer to a generic mmWave small cell operating in the Ka-band [18] wherein full-duplex (FD) paradigm has been additionally considered [17] to enhance the spectral efficiency. Key motivating factors for such a consideration are the short distances and low power which allow for the use of demonstrated technologies to mitigate self-interference [12].

While several options for enabling FD exist in small cells [17], we focus on the FD relaying scenario where the small cell BS acts as a FD relay forwarding the uplink signal from a user terminal (UT) onto the downlink of another UT on the same frequency. While the small-cell BS is FD enabled, the UTs operate in half-duplex mode.

\section{B. Scenario Description}

An illustration of the considered architecture is presented in Fig. 1. The outdoor small cells and the satellite gateway (GW) can be geographically separated and tools provided in the paper can be used to determine the exclusion zone [6], [3] for deployment of small cells in the vicinity of GW. For simplicity, the users are assumed to be uniformly distributed in the cells and we only depict the co-channel interference between a representative small cell and the GW. Further, the self-interference (SI) arising out of FDR is also illustrated. Furthermore, for the envisaged relaying scenario, the direct path between UT1 and UT2 is assumed to be absent.

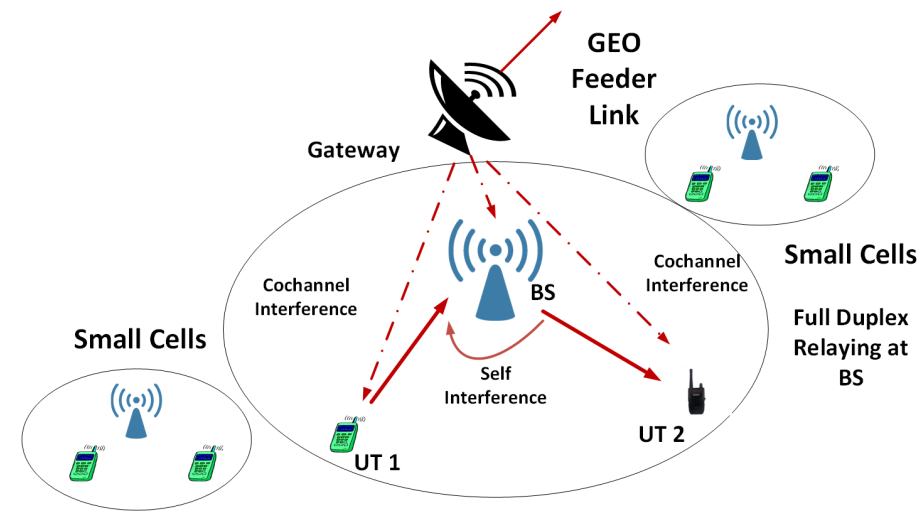

Fig. 1. Feeder link transmissions and Terrestrial Full Duplex Relaying
The FDR set-up discussed in Section II-A implies transmissions from UT1 to UT2 through the BS and those from UT2 to UT1 occur on different frequencies (half-duplex UT). Hence it suffices to depict only one path in Fig. 1.

\section{Signal Model}

To focus on the effects of co-existence and SI, we assume a common node of small cells that schedules transmissions to avoid inter-cell or multiuser interference. This allows us to consider communications within a single cell. For this setting, we now present the assumed signal model at various entities.

1) Satellite System: Since we consider a high powered satellite uplink and low powered small cells, we can safely assume the absence of interference at the GEO satellite receiver. Further, GW reception is unaffected by the small cell transmissions, since there is no reuse of the feeder downlink.

2) Small Cell BS: We consider a simplified processing at the BS involving only the signal amplification and addition of transmitter noise. This chain is representative of the considered relaying operation. While the Solid State Power Amplifiers (SSPA) are non-linear at higher efficiencies, we assume the use of predistortion techniques [20] to counter the non-linear effects. This allows us to consider linear processing at the BS. Further, we model SI as a scaled and delayed feedback of the BS output to its input. Such a model ensures a delay-free feedback loop as is the case in practical SI generation. Due to the assumed linear BS processing, we omit the non-linear terms in SI modelling.

3) Received Signal at UT2: The channels from the UT1 to BS and BS to UT2 are denoted respectively by $h_{B 1}$ and $h_{B 2}$, whose form is determined by the propagation impairments. For example, line-of-sight channels can be modelled as $h_{B 1}=$ $c G_{1} d_{1}^{L_{1}}$ and $h_{B 2}=c G_{2} d_{2}^{L_{2}}$, where the variables $G, d$ and $L$ denote antenna gain, distance, path loss exponent, respectively and $c$ is constant. With the aforementioned modelling, the expression for the signal received at UT2 then takes the form,

$$
\begin{aligned}
r_{B}[i]=h_{B 1} x_{1}[i]+h_{S I} t_{B}[i-\tau]+n_{R}[i]+I_{S B}[i], & \mathrm{UT} 1 \rightarrow \mathrm{BS}, \\
t_{B}[i]=\beta r_{B}[i]+n_{T}[i], & \mathrm{BS} \text { processing, } \\
y_{2}[i]=h_{B 2} t_{B}[i]+I_{S U}[i]+n_{U}[i], & \mathrm{BS} \rightarrow \mathrm{UT} 2,
\end{aligned}
$$

where, $x_{1}[i]$ is the transmitted signal from UT1 at instance $i, n_{R}[i]$ is the front-end receiver noise of the BS and $r_{B}[i]$ is the received signal at the BS. Equation (2) indicates the simplified gain plus noise model of the BS with $n_{T}[i]$ being the transmitter noise component. The scaling factor $\beta$ is the amplification gain and is chosen to ensure $E\left[\left|t_{B}[i]\right|^{2}\right]=P_{S}$, where $P_{S}$ is the BS transmit power and $E[\cdot]$ refers to the Expectation operator. The term $h_{S I}$ governs the power of SI leaking into the system while $\tau$ indicates the delay. Further, the signal received by UT2 is depicted using $y_{2}[i]$ with $n_{2}[i]$ being the UT2 receiver noise. The noise components $n_{R}[i], n_{T}[i]$ and $n_{U}[i]$ are modelled as i.i.d white Gaussian noise components with variance $\sigma_{R}^{2}, \sigma_{T}^{2}$ and $\sigma_{U}^{2}$ respectively. 
a) Interference from $G W$ : The terms $I_{S B}[i]$ and $I_{S U}[i]$, respectively, denote the interference from the satellite $\mathrm{GW}$ to the BS and the UT2. These are dependent on (a) distance of the BS (UT2) from the GW, (b) relative off-axis angles of the BS (UT2) and GW and (c) propagation impairments and antenna gains [3].

b) Internode Interference (INI): In a generic setting, UT2 suffers INI from UT1 transmissions in small cells [19]. However, in a FDR set-up, INI manifests as a direct path between the UTs. Since the scenario does not envisage a direct path, INI is omitted ${ }^{2}$.

\section{INTERFERENCE ANALYSIS}

We now analytically determine the contribution of various impairments, on the received SINR based on the models in (1)-(3). When $\left|h_{S I} \beta\right|<1$, exploiting (1) and (2), and substituting the resulting expression for $t_{B}[i]$ in (3), we get,

$y_{2}[i]=\beta h_{B 2} \sum_{j=0}^{\infty}\left(h_{S I} \beta\right)^{j}\left(h_{B 1} x_{1}[i-j \tau]+n_{R}[i-j \tau]\right)+I_{S U}$

$+n_{U}[i]+h_{B 2} \sum_{j=0}^{\infty}\left(h_{S I} \beta\right)^{j}\left(n_{T}[i-j \tau]+\beta I_{S B}[i-j \tau]\right)$.

With (4), we can identify the following:

Desired signal, $I_{d e s}$ : Obtained by scaling $x_{1}[i]$ as, $I_{d e s}=$ $\beta h_{B 1} h_{B 2} x_{1}[i]$. Clearly, $E\left[\left|I_{d e s}\right|^{2}\right]=\left|\beta h_{B 1} h_{B 2}\right|^{2} E\left[\left|x_{1}[i]\right|^{2}\right]$

Self Interference, $I_{S I}$ : For the chosen model, SI contains linear terms are given by, $I_{S I}=$ $\beta h_{B 1} h_{B 2} \sum_{l=1}^{\infty}\left(\beta h_{S I}\right)^{l} x_{1}[i-l \tau]$. Clearly, SI causes inter-symbol interference at the receiver and its power is $E\left[\left|I_{S I}\right|^{2}\right]=\left|\beta h_{B 2} h_{B 1}\right|^{2} \frac{\left|\beta h_{S I}\right|^{2}}{1-\left|\beta h_{S I}\right|^{2}} E\left[|x[i]|^{2}\right]$.

Noise Component, $I_{\eta}$ : This component comprises noise introduced by the BS and the front-end noise of the UT receiver. The effect of SI is not included and $I_{\eta}$ takes the form, $I_{\eta}=\beta h_{B 2} n_{R}[i]+h_{B 2} n_{T}[i]+n_{U}[i]$. The power of the noise component is $E\left[\left|I_{\eta}\right|^{2}\right]=\left|h_{B 2}\right|^{2}\left[|\beta|^{2} \sigma_{R}^{2}+\sigma_{T}^{2}\right]+\sigma_{U}^{2}$.

Full-Duplexing Noise, $I_{\gamma}$ : This term arises due to the interaction of the SI phenomenon with the noise components $n_{R}[i]$ and $n_{T}[i]$. It takes the form, $I_{\gamma}=$ $h_{B 2} \sum_{l=1}^{\infty}\left(\beta h_{S I}\right)^{l}\left(\beta n_{R}[i-l \tau]+n_{T}[i-j \tau]\right)$. Clearly, $I_{\gamma}=$ 0 in the absence of SI (i.e, $h_{S I}=0$ ) and its power is $E\left[\left|I_{\gamma}\right|^{2}\right]=\left|h_{B 2}\right|^{2} \frac{\left|\beta h_{S I}\right|^{2}}{1-\left|\beta h_{S I}\right|^{2}}\left[|\beta|^{2} \sigma_{R}^{2}+\sigma_{T}^{2}\right]$.

Co-channel Interference, $I_{C C I}$ : This component arises from the reuse of satellite frequency in terrestrial operations. Similar to the noise component, we do not include the effect of SI. We then have, $I_{C C I}=\beta h_{B 2} I_{S B}[i]+I_{S U}[i]$ and $E\left[\left|I_{C C I}\right|^{2}\right]=\left|\beta h_{B 2}\right|^{2} E\left[\left|I_{S B}[i]\right|\right]^{2}+E\left[\left|I_{S U}[i]\right|\right]^{2}$

Full-Duplexing CCI, $I_{\nu}$ : This term arises due to the interplay of SI and frequency reuse. In fact, $I_{\nu}=\beta h_{B 2} \sum_{l=1}^{\infty}\left(\beta h_{S I}\right)^{l} I_{S B}[i-l \tau]$ and $E\left[\left|I_{\nu}\right|^{2}\right]=\left|\beta h_{B 2}\right|^{2} \frac{\left|\beta h_{S I}\right|^{2}}{1-\left|\beta h_{S I}\right|^{2}} E\left[\left|I_{S B}[i]\right|^{2}\right]$.

\footnotetext{
${ }^{2}$ When the direct link is present, it can actually be exploited to enhance signal quality and then INI is no longer an interference.
}

Evaluating $\beta$ : We set $\beta$ to ensure $E\left[\left|t_{B}[i]\right|^{2}\right]=P_{S}$, where $P_{S}$ is the BS output power. Towards this, assuming statistical independence between desired signal, noise and satellite interference (SI and $I_{S B}[i]$ ), we get the following after some algebra,

$$
\beta^{* 2}=\frac{P_{S}-\sigma_{T}^{2}}{\left|h_{B 1}\right|^{2} P_{G}+\sigma_{R}^{2}+E\left[\left|I_{S B}[i]\right|^{2}\right]+P_{S}\left|h_{S I}\right|^{2}} .
$$

Assuming $h_{B 1}, h_{S I}, h_{B 2}$ are known at UT2 through prior calibration, we define the resulting SINR as,

$$
S I N R \triangleq \frac{E\left[\left|I_{d e s}\right|^{2}\right]}{E\left[\left|y_{2}[i]-I_{d e s}\right|^{2}\right]}
$$

Using $E\left[\left|x_{1}[i]\right|^{2}\right]=P_{G}$, a number of components from the denominator of (6) can be evaluated as discussed above. However, the interference components from the satellite, $I_{S B}, I_{S U}$ depend on a number of factors as described in Section II-C3a. Hence, obtaining a closed-form expression for (6) is difficult and we resort to simulations for evaluating it.

\section{Simulation Results and Discussions}

\section{A. Set-up}

We now numerically evaluate the feasibility of co-existence of BSS feeder uplinks and FDR small cells. The GW location is chosen as Betzdorf (Luxembourg), the GEO satellite is located at $28.2^{\circ} \mathrm{E}$ and the $\mathrm{BS}$ is located at $d=7 \mathrm{~km}$ from Betzdorf. We assume a small cell of radius of 200 metres with the BS located in the centre of the assumed circular coverage with line-of-sight channels for simplicity. Towards ease of implementation, we consider the worst-case scenario (from an interference point of view) where the offaxis angle between the GW and BS is chosen as the elevation angle at Betzdorf towards the desired satellite [3]. Towards determining the feasibility of the planned system, we evaluate the SINR in (6) for 10000 random user positions within the cell. Several quantities in (6) are determined using standard link budget analyses and Table I provides example values for the parameters needed to evaluate the link budgets.

The developed simulator takes in the user location and provides interference levels from the GW $\left(E\left[\left.I_{S B}[i]\right|^{2}\right], E\left[\left.I_{S U}[i]\right|^{2}\right]\right)$ using standard link-budget analysis [21]. Equation (1) indicates that the received power at BS (in the absence of SI and noise) is $\left|h_{B 1}\right|^{2} P_{G}$. Equating this to the received power at $\mathrm{BS}$ predicted by link analysis ${ }^{3}$ yields the values for $h_{B 1}$ (and $h_{B 2}$ similarly). Typically, we have $\left|h_{B 1}\right|^{2} \sim-85$ (in $\left.\mathrm{dB}\right)$ and $h_{B 2} \sim 71$ (in dB) ${ }^{4}$. Further, $\sigma_{R}^{2}, \sigma_{U}^{2}$ can be obtained from the typical noise figures of the $\mathrm{BS}$ and the UT respectively. We choose $\sigma_{T}^{2}=0.1 \sigma_{R}^{2}$.

\footnotetext{
${ }^{3}$ Received power $=$ Transmit EIRP - Free Space \& Propagation Losses + Receive antenna gain

${ }^{4}$ Small variations occur due to positions UT and BS
} 
TABLE I

GW PARAMETERS

\begin{tabular}{c|c}
\hline Parameter & Value \\
\hline Envisaged carrier frequency & $28 \mathrm{GHz}$ \\
Coexistence Bandwidth & $5 \mathrm{MHz}$ \\
GW EIRP & $82 \mathrm{dBW}$ \\
GW elevation & $29.3^{\circ}$ \\
GW Gain (off-axis) & $-4 \mathrm{~dB}$ \\
BS Antenna & $120^{\circ} \mathrm{Sector}$ \\
BS Antenna Gain & $17 \mathrm{dBi}$ \\
BS EIRP & $47 \mathrm{dBm}$ \\
BS Transmit Power $\left(P_{S}\right)$ & $30 \mathrm{dBm}$ \\
BS Noise Figure & $4 \mathrm{~dB}$ \\
UT EIRP & $20 \mathrm{dBm}$ \\
UT Antenna & isotropic \\
UT Antenna Gain & $0 \mathrm{dBi}$ \\
UT Noise Figure & $10 \mathrm{~dB}$ \\
UT Transmit Power $\left(P_{G}\right)$ & $20 \mathrm{dBm}$ \\
\hline
\end{tabular}

\section{B. Contributions of different interference components}

Fig. 2 depicts the contribution of different interference components to the total interference at UT2 for different values of SI power. The plot is obtained by evaluating the different interference terms as in Section III and normalizing them by $E\left[\left|I_{d e s}\right|^{2}\right]$. While $I_{\eta}$ and $I_{C C I}$ are known to be independent of $h_{S I}$, SI introduced components $E\left[\left|I_{S I}\right|^{2}\right], E\left[\left|I_{\gamma}\right|^{2}\right], E\left[\left|I_{\nu}\right|^{2}\right]$ depict an increase with SI power. This plot clearly shows that $E\left[\left|I_{S I}\right|^{2}\right.$ dominates the other components and that the cochannel interference from satellite $\mathrm{GW}$ is negligible for the considered scenario.

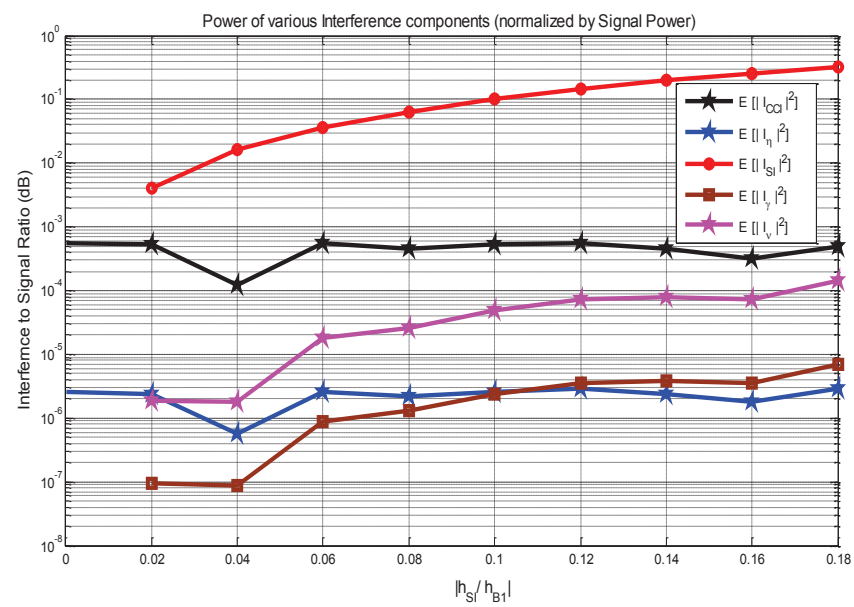

Fig. 2. Power of Interference components normalized by Signal power Interference to Signal Ratio

\section{Figure of Merit and Reference System}

Since the small-cell system is affected by the interference from the satellite uplink, we consider the spectral efficiency of small-cells, as the figure of merit. This figure of merit is simply,

$$
\eta=\frac{T_{t}}{B}
$$

where $T_{t}$, denotes the end-to-end throughput (in bits/ second) for the small cells and $B$ is the bandwidth used by the small cells. We now specialize $\eta$ for two different scenarios.

Reference System: This involves the satellite and smallcell coexistence scenario with traditional half-duplex operations at the UTs and the small-cell BS. In particular, we assume the reuse of feeder link frequencies in the small cells. We further assume that the $B \mathrm{~Hz}$ of bandwidth is divided equally for UT1 to BS and BS to UT2 transmissions. Letting each link of the half-duplex relaying small cells to have an efficiency of $R_{h d, t} \mathrm{~b} / \mathrm{s} / \mathrm{Hz}$, we have, $T_{t}=\frac{R_{h d, t} B}{2}$ and (7) yields $\eta_{\text {ref }}=\left(R_{h d, t}\right) / 2$.

Proposed System: This involves the proposed coexistence scenario with full-duplex operations at UTs, leading to use of $B \mathrm{~Hz}$ of bandwidth for UT1 to BS and BS to UT2 transmissions. Letting the full-duplex efficiency of small cells to be $R_{f d, t} \mathrm{~b} / \mathrm{s} / \mathrm{Hz}$ (when satellite frequencies are reused), we have, $T_{t}=R_{f d, t} B$ and we have $\eta_{p r o p}=R_{f d, t}$.

In the ensuing exercise, we obtain the spectral efficiency, $R_{*, *}$ for different systems by averaging the standard Shannon formula, $R_{*, *}=\log _{2}(1+S I N R)$, over UT locations after inserting appropriate values of end-to-end SINR.

\section{Gains in Spectral Efficiency}

Subsequent to computing the SINR for each user position, the achievable rate, $\log _{2}(1+S I N R)$, is averaged over 10000 positions. Fig. 3 depicts the achieved system spectral efficiency (in $\mathrm{b} / \mathrm{s} / \mathrm{Hz}$ ) in the small cells. It quantifies the gains when fullduplex cells are employed; it indicates that significant gains can be achieved if the SI component is low. In fact, gains for the full-duplex enabled system are obtained as along as $h_{S I} \leq 0.05 h_{B 1}$; using the approximate value of $h_{B 1}$ from Section IV-A, we can calculate the $\left|h_{S I}\right|^{2}$ to be approximately be $-110 \mathrm{~dB}$.

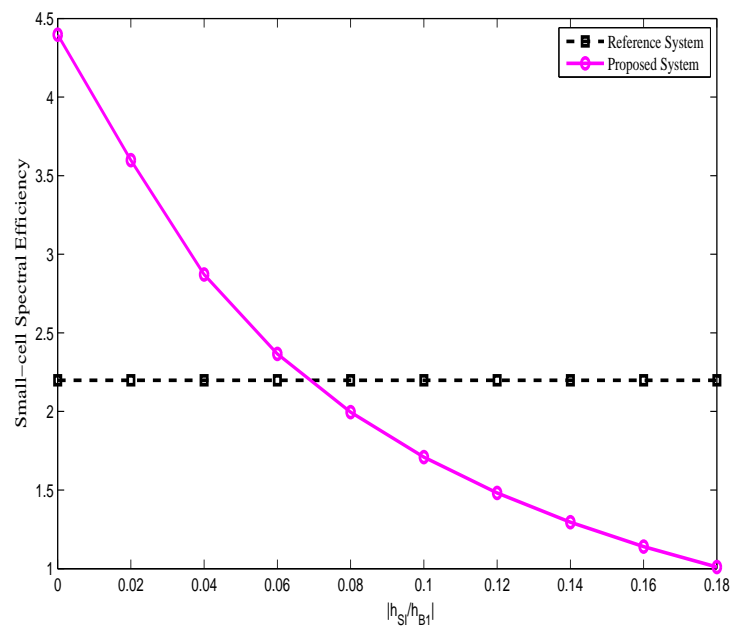

Fig. 3. Achievable small-cell spectral efficiency with reuse of satellite frequency 


\section{E. A note on interference mitigation}

The signal quality at the receiver is impacted by BS noise, interference from satellite $\mathrm{GW}$ and their interplay with the SI generation mechanism. Typically the feeder link transmissions use a different format and their interference cannot be cancelled. On the other hand, reducing SI serves to enhance the system performance. It can be identified from (1) that the term governing the SI power is $h_{S I}$. In an absolute sense, the current work can be seen as establishing limits on the maximum SI power for achieving gains over the reference system. On the other hand, the term $h_{S I}$ can also be viewed as a residual channel after incorporating the SI cancellation techniques; in this paradigm, the current work can be construed as a sensitivity analysis of the SI cancellers. With regards to the earlier example, the SI mitigation methods should ensure that the residual SI power $\left(\left|h_{S I}\right|^{2}\right)$ shall not exceed about $-110 \mathrm{~dB}$ (kindly refer Fig. 3); we are aided in the process by the directive mmWave transmissions.

Several techniques to mitigate the SI term $I_{S I}$ have been considered in literature [10] - [15]. In addition to SI cancellation at the BS, pre and post processing at UTs to mitigate SI can provide additional SI mitigation benefits. This is motivated by the fact that (3) indicates the SI in the received signal to cause inter-symbol interference. However, the purpose of the paper is to study the feasibility of extracting gains from the proposed setting; hence devising techniques for effecting SI cancellation is left for future investigation.

\section{CONCLUSIONS}

This paper proposed the reuse of satellite feeder link frequencies within full-duplex enabled small cells in the Kaband. Various impairments were modelled and SINR at a terminal was analytically derived to evaluate the impact of interference from full-duplex as well as reuse of feeder link frequencies. Analysis and performance evaluations depicted SI to be the dominating interference source while the contribution of the incumbent satellite GW is negligible. Further, fullduplexing was shown to enhance efficiency of current halfduplex systems when self-interference is sufficiently mitigated. Furthermore, reuse of frequencies provides additional gain due to lower co-channel interference. These observations further motivate the coexistence of satellite feeder uplinks (like BSS) and mmWave terrestrial small cells.

\section{REFERENCES}

[1] S. Maleki, S. Chatzinotas, B. Evans, K. Liolis, J. Grotz, A. VanelliCoralli, N. Chuberre, "Cognitive Spectrum Utilization in Ka Band Multi- beam Satellite Communications," IEEE Commun. Magazine., vol.53, no.3, pp.24-29, March 2015.

[2] S. Kandeepan, L. De Nardis, M. Di Benedetto, A. Guidotti, G.E. Corazza, "Cognitive Satellite Terrestrial Radios," IEEE Global Telecommunications Conference (GLOBECOM 2010), pp.1,6, 6-10 Dec. 2010.

[3] S. Maleki, S. Chatzinotas, J. Krause, K. Liolis, B. Ottersten, "Cognitive Zone for Broadband Satellite Communications in $17.317 .7 \mathrm{GHz}$ Band," IEEE Wireless Commun. Letters, vol.4, no.3, pp.305-308, June 2015.

[4] F. Guidolin, M. Nekovee, L. Badia, M. Zorzi, "A study on the coexistence of fixed satellite service and cellular networks in a mmWave scenario," IEEE International Conference on Communications (ICC), pp.24442449, 8-12 June 2015.

[5] R. Suffritti, G. E. Corazza, A. Guidotti, V. Petrini, D. Tarchi, A. VanelliCoralli, M. Di Renzo. "Cognitive hybrid satellite-terrestrial systems," Proceedings of the 4th International Conference on Cognitive Radio and Advanced Spectrum Management. ACM, 2011.

[6] M. Hoyhtya, J. Kyrolainen, A. Hulkkonen, J. Ylitalo, A. Roivainen, "Application of cognitive radio techniques to satellite communication," IEEE International Symposium on Dynamic Spectrum Access Networks (DYSPAN), pp.540-551, 16-19 Oct. 2012

[7] Small Cell Forum White Paper "Small cells - what's the big idea ?," 15 February 2012.

[8] T. Nakamura, S. Nagata, A. Benjebbour, Y. Kishiyama, T. Hai, S. Xiaodong, Y. Ning, L. Nan,“ Trends in small cell enhancements in LTE Advanced," IEEE Commun. Magazine, vol. 51, issue 2, pp. 98-105.

[9] DUPLO: Full-Duplex Radios for Local Access, Website :http://www.fp7-duplo.eu.

[10] B. P. Day, A. R. Margetts, D. W. Bliss, P. Schniter, "Full-duplex MIMO relaying: achievable rates under limited dynamic range," IEEE J. Select. Areas Commun., vol. 30, no. 8, pp. 1541-1553, Sept. 2012.

[11] A. Sabharwal, P. Schniter, D. Guo, D. Bliss, S. Rangarajan, R. Wichman, "In-band full-duplex wireless: challenges and opportunities," IEEE $J$. Sel. Area Commun., vol. 32, no. 9, pp. 1637-1652, Sept. 2014.

[12] M. Duarte, C. Dick, A. Sabharwal, "Experiment-Driven Characterization of Full-Duplex Wireless Systems," IEEE Trans. Wireless Commun., vol. 11, no. 12, pp. 4296-4307, Dec. 2012.

[13] D. Bharadia, E. McMilin, S. Katti, "Full duplex radios," in Proc. ACM Special Interest Group on Data Communication (SIGCOMM), Hong Kong, China, Aug. 2013, pp. 375-386.

[14] E. Everett, A. Sahai, A. Sabharwal, "Passive self-interference suppression for FD infrastructure nodes," IEEE Trans. Wireless Commun., vol. 13, no. 2, pp. 680-694, Jan. 2014.

[15] D. Bharadia, S. Katti, "Full duplex MIMO radios," in 11th USENIX Symp. on Networked Systems Design and Implementation (NSDI 14), Seattle, WA, April, 2014.

[16] Bhavani Shankar M. R., G. Zheng, S. Maleki B. Ottersten, "Feasibility Study of Full-duplex Relaying in Satellite Networks," Proceedings of 16th IEEE SPAWC, June 2015, Stockholm.

[17] Visa Tapio (editor) "System Scenarios and Technical Requirements for Full-Duplex Concept," DUPLO Deliverable D1.1, available online http://www.fp7-duplo.eu/index.php/deliverables,

[18] T. S. Rappaport, et. al, "Millimeter wave mobile communications for 5G cellular: It will work!," IEEE Access 1, 2013, pp. 335-349.

[19] S. Goyal, P. Liu, S. S. Panwar, R. A. Difazio, et. al "Full duplex cellular systems: will doubling interference prevent doubling capacity?," IEEE Commun. Magazine, vol. 53, issue 5, 2015, pp. 121-127.

[20] R. Piazza, Bhavani Shankar M. R, and B. Ottersten, "Carrier Rate Optimization on the Return Link of Interactive Mobile Satellite Networks,"in 20th European Wireless Conference, Barcelona, Spain, May, 2014.

[21] G. Maral and M. Bousquet, "Satellite Communication Systems: Systems, Techniques and AU14 Technologies," fourth ed., Wiley Eastern, 2002. 